\title{
The ultrasound-guided nerve blocks of abdominal wall contributed to anesthetic management of cholecystectomy in a patient with Becker muscular dystrophy without using muscle relaxants
}

\author{
Masato Iwata ${ }^{1 *}$, Naoya Kuzumoto', Yuka Akasaki', Masayo Morioka', Kana Nakayama', Nobuyoshi Matsuzawa', \\ Katsuhiro Kimoto ${ }^{2}$ and Toshiyuki Shimomura ${ }^{3}$
}

\begin{abstract}
Becker muscular dystrophy (BMD) is a progressive neuromuscular disorder caused by mutations in the dystrophin gene. The sensitivity to non-depolarizing muscle relaxant in a patient with muscle dystrophy is reportedly higher than that in normal individuals, and the duration of the effect is known to be prolonged. In this report, we present the case of a 58-year-old man with BMD who underwent laparoscopic cholecystectomy for symptomatic

cholelithiasis under total intravenous anesthesia without the use of muscle-relaxant drugs and supplemented with regional anesthesia. Anesthesia was induced and maintained with propofol, remifentanil, and fentanyl; ultrasoundguided bilateral rectus sheath block (RSB) and right-sided subcostal transversus abdominis plane block (TAP) were performed. The procedure required conversion to open surgery because of hard conglutination; intraoperative and postoperative periods were uneventful. Adequate analgesia was maintained after extubation because of the effect of RSB and TAP.
\end{abstract}

Keywords: Becker muscular dystrophy, Total intravenous anesthesia, Rectus sheath block

\section{Background}

Becker muscular dystrophy (BMD) is a relatively rare form of muscular dystrophy that has an incidence rate of approximately 1 in 18,000 live male births and a prevalence of $23.8 \times 10^{-6}[1]$. The condition results from mutations in the dystrophin gene [2] and is characterized by progressive weakness and wasting, predominantly of the proximal musculature. Patients with BMD undergoing surgery are susceptible to various intraoperative complications, such as aspiration pneumonia, prolonged artificial ventilation, malignant hyperthermia $(\mathrm{MH})$, rhabdomyolysis, hyperkalemia, cardiomyopathy, hypotension, and arrhythmia $[1,3]$.

The use of non-depolarizing muscle relaxants in these patients is associated with an increase in the maximal

\footnotetext{
*Correspondence: miwata@naramed-u.ac.jp

'Department of Anesthesiology, Nara Prefecture general Medical Center,

30-1, Hiramatsu 1-chome, Nara city, Nara 631-0846, Japan

Full list of author information is available at the end of the article
}

effect and duration of action [1]. Owing to the risk of malignant hyperthermia and adverse reactions to volatile anesthetics, total intravenous anesthesia (TIVA) is preferred in these patients [1]. Rectus sheath block (RSB) is known to induce abdominal relaxation $[4,5]$. In addition, RSB has been shown to reduce postoperative pain [5]. In our hospital, we perform RSB and rightsided subcostal transversus abdominis plane block to provide analgesia for laparoscopic cholecystectomy.

In this report, we present our experience with a case of BMD who underwent laparoscopic cholecystectomy under anesthesia with TIVA and RSB and without the use of muscle-relaxant drugs.

\section{Case presentation}

A 58-year-old man (height $166 \mathrm{~cm}$ and weight $75 \mathrm{~kg}$ ) was scheduled to undergo laparoscopic cholecystectomy for symptomatic cholelithiasis. He was diagnosed with BMD 
at 12 years. He had difficulty in walking because of weakness in leg muscles. Pulmonary function tests revealed a forced vital capacity of $50 \%$ of the predicted levels and forced expiratory volume in $1 \mathrm{~s}$ of $92 \%$ of predicted levels. He had not experienced any episode of cardiac failure; his electrocardiogram was almost normal.

General anesthesia was induced with the effect site concentration at $4 \mu \mathrm{g} / \mathrm{mL}$ propofol using a target-controlled infusion (TCI) pump (Terufusion TE-371, Terumo, Tokyo, Japan), $0.2 \mathrm{mg}$ fentanyl, and $0.25 \mu / \mathrm{kg}$ min remifentanil. Rocuronium was not used. He was maintained on $2.5-3 \mu \mathrm{g} / \mathrm{mL}$ propofol with TCI and $0.1-0.15 \mu \mathrm{g} / \mathrm{kg}^{\prime}$ min remifentanil with oxygenair mixture. Anesthesia was maintained under TIVA without the use of muscle relaxants. After induction, images of the rectus abdominis muscle were monitored with LOGIQ $\mathrm{e}^{\circ}$ (GE healthcare, Tokyo, Japan). Using a 20-G Tuohy needle (80 mm Hakko medical, Nagano, Japan), we performed ultrasound-guided bilateral RSB and right-sided subcostal TAPB with $0.75 \%$ ropivacaine $20 \mathrm{~mL}$ and $1 \%$ lidocaine $20 \mathrm{~mL}$, for abdominal relaxation.

Mid-way through the surgery, the procedure required conversion to open surgery because of hard conglutination; however, non-depolarizing muscle relaxants were not required. As an analgesic, $1000 \mathrm{mg}$ of acetaminophen was administered. After the completion of surgery, he was extubated and the patient did not feel any pain because of the wound.

For pain relief, flurbiprofen axetil $50 \mathrm{mg}$ div was medicated after he was transferred to the surgical ward, after 8 , and $16 \mathrm{~h}$ from the operation. Numerical Rating Scale regarding the wound pain changed $4 \sim 6$ on the surgery day and $2 \sim 3$ on postoperative day 1 . Postoperative complication of bile leak afflicted him, but on postoperative day 93 , the patient was discharged.

\section{Discussion}

We reported that bilateral rectus sheath block and rightsided subcostal transversus abdominis plane block contributed to successful anesthetic management of laparoscopic cholecystectomy, conversed to open cholecystectomy under total intravenous anesthesia without the use of muscle relaxants, in a patient with BMD. Because of the involvement of complex multisystems in BMD patients undergoing surgery, several specific perioperative considerations may significantly impact on surgical outcomes $[1,2]$. Several reports on the management of anesthesia in BMD patients are available in the published literature [1-3]. Up to 50$70 \%$ patients with muscular dystrophy manifest cardiac abnormalities, although it is clinically significant in only $10 \%$ of these patients [6]. An estimated 30\% of deaths in individuals with Duchenne muscular dystrophy (DMD) are due to respiratory causes. Therefore, careful preoperative pulmonary assessment is of critical importance. Reports have suggested a relationship between DMD or BMD and MH. Use of volatile anesthetics in patients with muscular dystrophy is associated with an increased risk of rhabdomyolysis, which could result in cardiac arrest and $\mathrm{MH}$ [3]. Therefore, volatile anesthetic agents should be avoided and TIVA is recommended in these patients [7].

Limited use of non-depolarizing muscle relaxants may be allowed depending on situations in consideration of increased maximal effect and duration of action [8]. However, a literature is available that reports the safe use of sugammadex to antagonize the neuromuscular block and rapid recovery in DMD [9].

\section{Conclusions}

In this report, we present a case of BMD in whom laparoscopic cholecystectomy was performed under TIVA and RSB and without the use of muscle-relaxant drugs; good postoperative analgesia was achieved with this regime.

\section{Authors' contributions}

$\mathrm{Ml}$ collected the data and drafted the manuscript. NK, YA, MM, KN, NM, KK, and TS revised the manuscript. All authors read and approved the final manuscript for submission.

\section{Consent for publication}

Written informed consent was obtained from the patient for publication of this case report.

\section{Competing interests}

The authors declare that they have no competing interests.

\section{Publisher's Note}

Springer Nature remains neutral with regard to jurisdictional claims in published maps and institutional affiliations.

\section{Author details}

${ }^{1}$ Department of Anesthesiology, Nara Prefecture general Medical Center, 30-1, Hiramatsu 1-chome, Nara city, Nara 631-0846, Japan. ${ }^{2}$ Department of Anesthesiology, Nara Medical University, Kashihara, Japan. ${ }^{3}$ Perioperative Care Center, Nara Prefecture General Medical Center, Nara, Japan.

Received: 28 October 2017 Accepted: 28 November 2017

Published online: 08 December 2017

\section{References}

1. Miller RD. Miller's anesthesia. 8th ed. Philadelphia: Saunders; 2015. p. 1302-6.

2. Chen HE, Cripe L, Tobias JD. Perioperative management of a patient with Becker's muscular dystrophy. Pediatr Anesthe Crit Care J. 2013;1:50-60.

3. Shimauchi T, Yamaura K, Sugibe S, Hoka S. Usefulness of sugammadex in a patient with Becker muscular dystrophy and dilated cardiomyopathy. Acta Anaesthesiol Taiwanica. 2014:52:146-8.

4. Schleich CL. Schmerzlose operationen. 4th ed. Berlin: Springer Verlag; 1899. p. $240-8$

5. Smith BE, Suchak M, Siggins D, Challands J. Rectus sheath block for diagnostic laparoscopy. Anaesthesia. 1988;43:947-8.

6. Urban M, Lahlou S. Muscle diseases. In: Fleisher L, editor. Anesthesia and uncommon diseases. Philadelphia: Saunders; 2006. p. 303.

7. Poole TC, Lim TY, Buck J, Kong AS. Perioperative cardiaC arrest in a patient with previously undiagnosed Becker's muscular dystrophy after isoflurane anaesthesia for elective surgery. Br J Anaesth. 2010;104:487-9.

8. Ririe DG, Shapiro F, Sethna NF. The response of patients with Duchenne's muscular dystrophy to neuromuscular blockade with vecuronium. Anesthesiology. 1998:88:351-4.

9. Wefki Abdelgawwad Shousha AA, Sanfilippo M, Sabba A, Pinchera P. Sugammadex and reversal of neuromuscular block in adult patient with duchenne muscular dystrophy. Case Rep Anesthesiol. 2014;2014:680568. 